\title{
TAME THEORIES WITH HYPERARITHMETIC HOMOGENEOUS MODELS
}

\author{
TERRENCE MILLAR
}

(Communicated by Thomas J. Jech)

\begin{abstract}
A tame theory is a decidable first-order theory with only countably many countable models, and all complete types recursive. It is shown here that the recursive complexity of countable homogeneous models of tame theories is unbounded in the hyperarithmetic hierarchy.
\end{abstract}

Recursive model theory considers model-theoretic objects and constructions from the point of view of recursion-theoretic complexity. For instance, must isomorphism types of countable models that are structurally simple have models of low recursion-theoretic complexity? This paper considers one such question involving countable homogeneous models. If $\mathfrak{C}$ is a countable homogeneous model of a complete theory $T$, then is $\mathfrak{C}$ necessarily decidable in the degree of $T$ ? Since there are complete theories with $2^{\omega}$ complete types, the answer is obviously "no". On the other hand, by results in [1], if $T$ has only finitely many countable models and all of the complete types of the theory are recursive, then the answer is "yes". This paper considers decidable theories $T$ that have at most countably many countable models and only recursive complete types. Call such a theory tame. The notational conventions in the paper are standard.

Fact. Every countable homogeneous model of a tame theory $T$ is hyperarithmetic.

Proof. Let $\left\{\mu_{i} \mid i<\omega\right\}$ be an effective enumeration of all the partial recursive functions $\mu: N \rightarrow N$, and let $\left\{\theta_{i} \mid i<\omega\right\}$ be an effective enumeration of all formulas in $L(T)$. Since all of the types of $T$ are recursive, we can fix a hyperarithmetic set $I \subseteq N$ such that

(i) $\forall i \in I\left[\left\{\theta_{j} \mid \mu_{i}(j)=1 ; j<\omega\right\}\right.$ is a complete type of $\left.T\right]$; and

(ii) $\forall \Gamma$ complete type of $T \quad \exists ! i \in I \quad \forall j\left[\theta_{j} \in \Gamma\right.$ iff $\left.\mu_{i}(j)=1\right]$.

For a countable model $\mathfrak{U} \vdash T$, say that $W \subseteq I$ is a witness set for $\mathfrak{U}$ iff there is some enumeration $\left\{\bar{a}_{i} \mid i<\omega\right\}$ of $|\mathfrak{U}|^{<\omega}$ such that

(1) $\forall n \in W\left[\mu_{n}\right.$ is total];

Received by the editors September 15, 1987.

1980 Mathematics Subject Classification (1985 Revision). Primary 03C57.

Research for this paper was partially supported by Australian Research Grant \#8215113 and NSF Grant \#DMS 8501521. 
(2) $\forall n \in W \exists i \forall j\left[\left\langle\mathcal{U}, \bar{a}_{i}\right\rangle \vDash \theta_{j}\left(\bar{a}_{i}\right)\right.$ iff $\left.\mu_{n}(j)=1\right]$;

(3) $\forall i \exists n \in W \forall j\left[\left\langle\mathcal{U}, \bar{a}_{i}\right\rangle \vDash \theta_{j}\left(\bar{a}_{i}\right)\right.$ iff $\left.\mu_{n}(j)=1\right]$.

Note that each model of $T$ has a witness set, and by choice of $I$, the witness set for a given model is unique. Therefore it is not difficult to see that the set of witness sets of models of $T$ is $\Sigma_{1}^{1}$. Since $T$ has only countably many type spectra, it follows that every model of $T$ has a hyperarithmetic witness set. Thus every countable homogeneous model of $T$ has a hyperarithmetic witness set. It follows by the results in [3] that every countable homogeneous model of $T$ is hyperarithmetic.

In [2] a tame theory was constructed such that the countable saturated model of the theory was not decidable. In that paper it was noted that the saturated model of such a theory must be decidable in $0^{\prime}$. The result of this paper is that there is no hyperarithmetic bound on the complexity of countable homogeneous models of tame theories. Two observations are perhaps useful to make. First, a homogeneous structure is model-theoretically simple enough that its isomorphism type is completely determined by the structure's cardinality and type spectrum. Second, by results in [3], a countable homogeneous model of a tame theory is decidable in the join of the degree of the type spectrum of that model and that of the type spectrum of the saturated model. Since the type spectrum of the saturated model of a tame theory is always $\Sigma_{2}^{0}$, the construction necessarily will involve coding recursion-theoretic complexity into the type spectrum of the desired model. The primary difficulty in producing the desired theory is controlling the forcing relationship between types. The types must be sufficiently independent to allow for a complex type spectrum for the desired model, and yet dependent enough to prevent uncountably many type spectra for different models of the theory.

Theorem. For every hyperarithmetic degree $A$ there is a tame theory that has a countable homogeneous model that is not decidable in $A$.

Proof. It is well known [4] that the Turing degrees that can be realized as the degree of a branch in a recursive tree $\operatorname{Tr} \subseteq 2^{<\omega}$ with at most countably many branches is unbounded in the hyperarithmetic hierarchy $\left[f \in 2^{\omega}\right.$ is a branch of $\operatorname{Tr}$ iff $\left._{\mathrm{df}} \forall n<\omega(f \mid n \in \operatorname{Tr})\right]$. So for an arbitrary hyperarithmetic degree $A$ fix such a tree $\operatorname{Tr}$ that has a branch $f$ not Turing reducible to $A^{\prime \prime}$. We will assume without loss that $\operatorname{Tr}$ has no terminal nodes. This tree will be coded into the type structure of the desired theory $T$. This will be done such that there is a countable $\mathfrak{B} \vdash T$ with the property that $f$ is recursive in $S^{\prime \prime}$, where $S$ is the satisfaction predicate for any model isomorphic to $\mathfrak{B}$. This will imply that $\mathfrak{B}$ is not decidable in $A$.

A nonprincipal 1-type will be associated with each node of the tree. This will be accomplished in such a way that the set of such types realized in the desired model is exactly the set of types associated with the nodes of the infinite branch $f$. In order to control the number of type spectra, two devices are employed. 
Let $\Gamma_{\xi}$ denote the type associated with the node $\xi$. For $\xi, \eta$ in the tree, if $\xi<\eta$ then it will be arranged that whenever $\Gamma_{\eta}$ is realized in a model, $\Gamma_{\xi}$ must also be realized. Notice that this strategy will not interfere with the first goal mentioned. This still does not prevent uncountably many type spectra, since, in the cases of interest, there will be an infinite set of pairwise incomparable nodes. The second strategy therefore will be aimed at antichains. Specifically, if $\xi$ and $\eta$ are in the tree, and are not comparable, then any model realizing both $\Gamma_{\xi}$ and $\Gamma_{\eta}$ will also be forced to realize $\Gamma_{\delta}$ for all $\delta$ in the tree whose length is less than or equal to that of $\xi$ or $\eta$.

Language for $T$ :

$$
\begin{array}{lll}
\text { constants: } & c_{\xi, i}, \quad \xi \in \operatorname{Tr}, i<\omega ; \\
\text { unary predicate: } & P ; \\
\text { binary predicates: } E,<; \\
\text { unary functions: } & F_{\xi, \eta}, \quad \xi<\eta \in \operatorname{Tr}, \\
& I_{\xi, \eta}, \quad \operatorname{lh}(\xi)=\operatorname{lh}(\eta)>0, \quad \xi, \eta \in \operatorname{Tr} ; \\
\text { binary functions: } & G_{\xi, \eta}, \quad \operatorname{lh}(\xi)=\operatorname{lh}(\eta)>0, \xi, \eta \in \operatorname{Tr}, \\
& g .
\end{array}
$$

$T$ will be the model completion of the universal theory $T^{\prime}$. The axioms for $T^{\prime}$ are the universal closures of the following [the convention will be that variable subscripts are to range over all possible values such that the corresponding symbols are in the language, subject to any additional constraints that are stated]:

$E$ is an equivalence relation:

1. $E(x, x)$;

2. $E(x, y) \rightarrow E(y, x)$;

3. $E(x, y) \wedge E(y, z) \rightarrow E(x, z)$.

$<$ is a tree order:

4. $\sim(x<x)$;

5. $x<y \wedge y<z \rightarrow x<z$

6. $x<z \wedge y<z \rightarrow x<y \vee y<x \vee x=y$.

$<$-related elements are equivalent:

7. $x<y \rightarrow E(x, y)$.

$g$ is a meet operator within each equivalence class:

8. $g(x, y)=g(y, x)$;

9. $z<x \wedge z<y \rightarrow z<g(x, y) \vee z=g(x, y)$;

10. $E(x, y) \rightarrow g(x, y)<x \vee g(x, y)=x$;

11. $\sim E(x, y) \rightarrow g(x, y)=c_{\langle\rangle, 0}$.

The $c_{\xi}$ 's inhabit distanct equivalence classes:

12. $E\left(c_{\xi, i}, c_{\xi, j}\right)$;

13. $\sim E\left(c_{\xi, i}, c_{\eta, j}\right) \quad \xi \neq \eta$.

The $c_{\xi, i}$ 's form an $\omega$-chain: 
14. $c_{\xi, i}<c_{\xi, i+1}$.

Realizations of $\sim P$ are <-incomparable and $P$ forms <-initial trees:

15. $x \neq y \rightarrow P(g(x, y))$.

Each $c$ is in $P$ :

16. $P\left(c_{\xi, i}\right)$.

The nontrivial domain of $F_{\xi, \eta}$ is the $c_{\xi}$-equivalence class; the range of $F_{\xi, \eta}$ is the $c_{\eta}$-equivalence class together with $c_{\langle\rangle, 0}$ :

17. $\sim E\left(c_{\xi, 0}, x\right) \rightarrow F_{\xi, \eta}(x)=c_{\langle\rangle, 0}$;

18. $E\left(c_{\xi, 0}, x\right) \rightarrow E\left(F_{\xi, \eta}(x), c_{\eta, 0}\right)$.

$F_{\xi, \eta}$ is <-order and $P$-preserving (but not meet-preserving):

19. $E\left(c_{\xi, 0}, x\right) \wedge x<y \rightarrow F_{\xi, \eta}(x)<F_{\xi, \eta}(y)$;

20. $F_{\xi, \eta}\left(c_{\xi, i}\right)=c_{\eta, i}$

21. $E\left(c_{\xi, 0}, x\right) \rightarrow\left[P(x) \leftrightarrow P\left(F_{\xi, \eta}(x)\right)\right]$.

$I_{\xi, \eta}$ is an isomorphism between $P$ parts of the $c_{\xi}$ and $c_{\eta}$ equivalence classes:

22. $\sim E\left(c_{\xi, 0}, x\right) \vee \sim P(x) \rightarrow I_{\xi, \eta}(x)=c_{\langle\rangle, 0}$;

23. $E\left(c_{\xi, 0}, x\right) \wedge P(x) \rightarrow E\left(I_{\xi, \eta}(x), c_{\eta, 0}\right) \wedge P\left(I_{\xi, \eta}(x)\right)$;

24. $\left[E\left(c_{\xi, 0}, x\right) \wedge E(x, y) \wedge P(x) \wedge P(y)\right] \rightarrow I_{\xi, \eta}(g(x, y))=g\left(I_{\xi, \eta}(x), I_{\xi, \eta}(y)\right)$;

25. $I_{\xi, \eta}\left(c_{\xi, i}\right)=c_{\eta, i}$.

The $F$ 's and the $I$ 's commute on subscripts:

26. $I_{\eta, \xi}\left(F_{\lambda, \eta}(x)\right)=F_{\delta \xi}\left(I_{\lambda, \delta}(x)\right)$.

The $I$ 's compose on subscripts:

27. $I_{\xi, \delta}\left(I_{\eta, \xi}(x)\right)=I_{\eta, \delta}(x)$;

28. $E\left(c_{\xi, 0}, x\right) \rightarrow I_{\xi, \xi}(x)=x$.

The $F$ 's compose on subscripts:

29. $F_{\xi, \delta}\left(F_{\eta, \xi}(x)\right)=F_{\eta, \delta}(x)$.

The non-trivial domain of $G_{\xi, \eta}$ is the cartesian product of the $\sim P$-parts of the $c_{\xi}$ and $c_{\eta}$ equivalence classes, and the range is the $P$-part of the $c_{\eta}$ equivalence class together with $c_{\langle\rangle, 0}$ :

30. $\sim E\left(c_{\xi, 0}, x\right) \vee \sim E\left(c_{\eta, 0}, y\right) \vee P(x) \vee P(y) \rightarrow G_{\xi, \eta}(x, y)=c_{\langle\rangle, 0}$;

31. $E\left(c_{\xi, 0}, x\right) \wedge E\left(c_{\eta, 0}, y\right) \wedge \sim P(x) \wedge \sim P(y) \rightarrow E\left(G_{\xi, \eta}(x, y), c_{\eta, 0}\right) \wedge$ $P\left(G_{\xi, \eta}(x, y)\right)$.

$G_{\xi, \eta}$ behaves like a greatest lower bound operator on its domain:

32. $E\left(c_{\xi, 0}, x\right) \wedge E\left(c_{\eta, 0}, y\right) \wedge \sim P(x) \wedge \sim P(y) \wedge z<y \wedge I_{\eta, \xi}(z)<x \rightarrow z \leq$ $G_{\xi, \eta}(x, y)$;

33. $E\left(c_{\xi, 0}, x\right) \wedge E\left(c_{\eta, 0}, y\right) \wedge \sim P(x) \wedge \sim P(y) \rightarrow G_{\xi, \eta}(x, y)<y$ $\wedge I_{\eta, \xi}\left(G_{\xi, \eta}(x, y)\right)<x$.

The verification that $T^{\prime}$ has a complete, decidable, model completion $T$ is physically at the end of this paper, in Lemma 10 . Since $T$ is the model completion of a universal theory, it allows elimination of quantifiers. Each model of $T$ has infinitely many distinct $E$-equivalence classes. There is a distinct $E$-equivalence class for each $c_{\xi, 0}, \xi \in \operatorname{Tr}$. Within each $E$-equivalence 
class, $<$ is a dense $\omega$-branching tree order. The realizations of $\sim P$ are $<-$ maximal elements, and so the realizations of $P$ are an initial tree within each equivalence class. In any model of $T$, and for any realization of $P$ in that model, there is a realization of $\sim P<-$ above it. Within the equivalence class of $c_{\eta, 0}, G_{\eta, \eta}$ and $g$ agree on pairs of $\sim P$ realizations. The domain of any function symbol $X_{\eta, \xi}$ is essentially the $E$-equivalence class of $c_{\eta, 0}$, and its range is essentially the $E$-equivalence class of $c_{\xi, 0}$. The $I$ 's provide an isomorphism between $P$-realizations in the $E$-equivalence classes of $c_{\eta, 0}$ and $c_{\xi, 0}$, for $\eta, \xi \in \operatorname{Tr}$ such that $\operatorname{lh}(\eta)=\operatorname{lh}(\xi)$. The $F$ 's do not commute with the meet operator $g$. This was intentional and allows the limit type associated with $\eta$ to be nonprincipal over the limit type associated with $\xi$, where $\xi<\eta$. On the other hand $F_{\eta, \xi}$ is order-preserving within the $c_{\eta, 0}$ equivalence class so that the limit type associated with $\xi$ is principal over the limit type associated with $\eta$. These remarks are made more precise in the lemmas that follow.

Lemma 1. The closure under the functions of any finite subset of a model of $T^{\prime}$ is finite.

Proof. We will use 'closure' for 'closure under the functions' in what follows. Fix $\mathfrak{C} \vDash T^{\prime}$ and $\bar{a} \in|\mathfrak{C}|^{<\omega}$. The proof is by induction on the length of the longest $\xi$ such that, for some $a \in \operatorname{rg}(\bar{a}), \mathfrak{C} \vDash E\left(c_{\xi, 0}, a\right)$.

If there is no such $\xi$ or $\xi=\langle\rangle$, then the lemma follows easily from the axioms in $8-11,17,22$, and 30 . Suppose the lemma is true for lengths less than $n>0$. Assume without loss that $a=\left\langle a_{0}, \cdots, a_{m}\right\rangle$ satisfies, for some $s$, $0<s<m$ :

(1) $\forall i \leq s$ [if $\mathfrak{C} \vDash E\left(c_{\xi, 0}, a_{i}\right)$ then $\operatorname{lh}(\xi)=n$ ];

(2) $\forall i>s$ [if $\mathbb{C} \vDash E\left(c_{\xi, 0}, a_{i}\right)$ then $\ln (\xi)<n$ ].

By the axioms in 15-16 and 30-33 the closure $\vec{a}^{\prime}$ of $\left\langle a_{0}, \cdots, a_{s}\right\rangle$ under the $G$ 's is finite. By the axioms in 8-11,22-24, 27, and 28 the closure $\bar{b}$ of $\bar{a}^{\prime}$ under $g$ and the $I$ 's is finite. By the same axioms, $\bar{b}$ is already closed under the $G$ 's. Also, by the axioms in 8-11, 22-23, and 30-34, for all $b \in \operatorname{rg}(\bar{b})$, if $\mathfrak{C} \vDash E\left(c_{\xi, 0}, b\right)$ then either $\xi=\langle\rangle$ or $\ln (\xi)=n$. It then follows from the axioms in 17-21 that for any $F_{\xi, \eta}, b \in \operatorname{rg}(\bar{b}), \delta \in \operatorname{Tr}$, if

$$
\mathfrak{C} \vDash E\left(c_{\delta, 0}, F_{\xi, \eta}(b)\right),
$$

then $\operatorname{lh}(\delta)<n$. Thus, by the axioms in 11,22 , and 30 , the closure of $\operatorname{rg}(\bar{a})$ is equal to the union of $\operatorname{rg}(\bar{b})$ and the closure of $D$, where

$$
D=\left\{a_{i} \mid s<i<m\right\} \cup\left\{F_{\xi, \eta}(b) \mid b \in \operatorname{rg}(\bar{b}), \eta<\xi, \operatorname{lh}(\xi)=n\right\} .
$$

But the closure of $D$ is finite by the induction hypothesis.

A closed subset of a model of $T$ is a subset that is closed under the function symbols. Similarly, a complete type of $T$ is closed if it is realized by a closed subset of some model of $T$. 
Lemma 2. Let $L^{\prime}$ be the language of $T$ with the $F_{\xi, \eta}$ symbols removed. Each of the following sets of formulas uniquely determines a quantifier free 1-type of $\left.T\right|_{L^{\prime}}$ by its containment. Conversely, each quantifier free 1-type of $\left.T\right|_{L^{\prime}}$ contains exactly one of these sets of formulas:

(i) $\left\{\sim E\left(c_{\xi, 0}, x\right), P(x)^{k} \mid \xi \in \operatorname{Tr}\right\}, k<2$;

(ii) $\left\{c_{\xi, i}<x, P(x)^{k} \mid i<\omega\right\}, \xi \in \operatorname{Tr}, k<2$;

(iii) $\left\{c_{\xi, i}=x\right\}, \xi \in \operatorname{Tr}, i<\omega$;

(iv) $\left\{x<c_{\xi, i}, c_{\xi, i-1}<x\right\}, \xi \in \operatorname{Tr}, i<\omega$; or

(v) $\left\{E\left(x, c_{\xi, 0}\right),\left(c_{\xi, i-1}<x\right), \sim\left(c_{\xi, i} \leq x\right), \sim\left(x \leq c_{\xi, i}\right), P(x)^{k}\right\}, k<2$, $\xi \in \operatorname{Tr}, i<\omega$.

Proof. Let $\Gamma$ be a 1-type of $\left.T\right|_{L^{\prime}}$ that contains the set in (i). Then by the axioms in $1,4,9,10,22$, and 30 the result follows. The proof for (ii)-(v) also depend on the same set of axioms, as well as the axioms from 23-25, 27, 28, and $31-33$. The second assertion is easy to verify.

Denote the unique type containing a set from (i)-(v) above by, respectively:

(i) $\Gamma_{\omega, k}$;

(ii) $\Gamma_{\xi, k}$;

(iii) $\Gamma_{\xi, i,=}^{\zeta, k}$;

(iv) $\Gamma_{\xi, i,<}^{\xi, i,=}$

(v) $\Gamma_{\xi, i, k}^{\xi}$.

Lemma 3. If two 1-types of $T$ contain the same set of formulas from (i)-(iv) above, then they are equal.

Proof. This follows from elimination of quantifiers, Lemma 2, and the axioms in $17-21,26,28$, and $30-33$.

If a 1-type $\Sigma$ of $T$ contains $\Gamma_{\xi, j, k}$ then the trace of $\Sigma$ is defined to be the $\bar{u}$ such that, for $i<\ln (\xi)$ :

(i) $\ln (\bar{u})=\ln (\xi)+1$;

(ii) $\bar{u}(i)=\langle m, \mid\rangle$ iff $\Gamma_{\eta, m, k}\left(F_{\xi, \eta}(x)\right) \subseteq \Gamma_{\xi, j, k}(x)$, where $\eta=\left.\xi\right|_{i}$;

(iii) $\bar{u}(i)=\left\langle m, \Rightarrow\right.$ iff $\Gamma_{\eta, m,=}\left(F_{\xi, \eta}(x)\right) \subseteq \Gamma_{\xi, j, k}(x)$, where $\eta=\left.\xi\right|_{i}$;

(iv) $\bar{u}(i)=\langle m,<\rangle$ iff $\Gamma_{\eta, m,<}\left(F_{\xi, \eta}(x)\right) \subseteq \Gamma_{\xi, j, k}(x)$, where $\eta=\left.\xi\right|_{i}$;

(v) $\bar{u}(i)=\omega$ iff $\Gamma_{\eta, k}\left(F_{\xi, \eta}(x)\right) \subseteq \Gamma_{\xi, j, k}(x)$, where $\eta=\left.\xi\right|_{i}$;

(vi) $\bar{u}(\operatorname{lh}(\xi))=\langle\xi, k\rangle$;

Lemma 4. If $\bar{u}$ is the trace of $\Sigma \supseteq \Gamma_{\xi, j, k}$ then:

(i) if $\bar{u}(i)=\langle m, X\rangle$ and $\bar{u}(i-1)=\langle n, Y\rangle$, then $m \leq n$;

(ii) if $\bar{u}(i)=\langle m,=\rangle$ and $i\rangle 0$, then $\bar{u}(i-1)=\langle m,=\rangle$;

(iii) if $\bar{u}(i)=\langle m,<\rangle$ and $i\rangle 0$, then $\bar{u}(i-1)=\langle m,<\rangle$;

(iv) if $\bar{u}(i)=\omega$ and $i>0$, then $\bar{u}(i-1)=\omega$.

Proof. Each of these follow from some combination of the axioms in 13, 19, and 20. 
The characteristic of a 1-type $\Gamma$ is defined to be $\bar{v}$ such that:

(i) $\Gamma(x) \supseteq \Gamma_{\omega, k}(x)$ iff $\bar{v}=\langle\omega, k\rangle$;

(ii) $\Gamma(x) \supseteq \Gamma_{\xi, k}(x)$ iff $\bar{v}=\langle\xi, \omega, k\rangle$;

(iii) $\Gamma(x) \supseteq \Gamma_{\xi, j,=}(x)$ iff $\bar{v}=\langle\xi,=\rangle$;

(iv) $\Gamma(x) \supseteq \Gamma_{\xi, j,<}(x)$ iff $\bar{v}=\langle\xi,<\rangle$;

(v) $\Gamma(x) \supseteq \Gamma_{\xi, j, k}(x)$ iff $\bar{v}$ is the trace of $\Gamma(x)$.

The characteristic of an $n$-type $\Sigma(\bar{x}), n>1$, is the $\bar{w}$ such that

(i) $\ln (\bar{x})=\ln (\bar{w})$;

(ii) $\forall i<\ln (\bar{w})[\bar{w}(i)$ is the characteristic of the 1-type projection of $\Sigma(\bar{x})$ determined by $x_{i}$ ].

Lemma 5. (i) Each 1-type of $T$ has and is uniquely determined by a characteristic;

(ii) Every complete closed type $\Gamma\left(x_{1}, \cdots, x_{n}\right)$ of $T$ is uniquely determined by its 1-type projections and a finite subset that it contains form:

$$
\left\{\left(\tau_{1}=\tau_{2}\right)^{k},\left(\tau_{1}<\tau_{2}\right)^{k}, E\left(\tau_{1}, \tau_{2}\right)^{k} \mid k<2 ; \tau_{1}, \tau_{2} \text { terms in } x_{1}, \cdots, x_{n}\right\} \text {; }
$$

(iii) $T$ has only recursive complete types.

Proof. (i) follows routinely from Lemmas $2-4$ and their proofs. (ii) depends on the previous lemmas, the axioms for $T^{\prime}$, and the elimination of quantifiers. (iii) is then easily verified.

Lemma 6. (i) If $\xi<\eta \in \operatorname{Tr}$, then every model of $T$ realizing $\Gamma_{\eta, k}$ must also realize $\Gamma_{\xi, k}$;

(ii) Each of the following types is nonprincipal in the theory $\Gamma_{\eta, 1}(a)$ :

(a) $\Gamma_{\xi, 0}(x), \xi \in \operatorname{Tr}$;

(b) $\Gamma_{\xi, 1}(x), \xi \in \mathrm{Tr}$, not $\xi \leq \eta$;

(c) $\Gamma_{\omega, k}^{\xi x}(x), k<2$;

(iii) If $\xi, \eta \in \operatorname{Tr}$ and $\operatorname{Ph}(\xi)<\operatorname{Ph}(\eta)$, then every model realizing both $\Gamma_{\xi, 1}$ and $\Gamma_{\eta, 0}$ must also realize $\Gamma_{n, 1}$.

Proof. For (i) consider the formula $x=F_{\xi, \eta}(a)$. By the axioms in 14 and 18-20 it follows that

$$
\Gamma_{\eta}(z) \vdash \forall x\left[x=F_{\xi, \eta}(a) \rightarrow\left(c_{\xi, i}<x\right)\right], \quad i<\omega .
$$

This, with the axioms in 21 , establishes (i).

The proof of (ii) follows from elimination of quantifiers, the signature and axioms, and in particular the fact that $I_{\nu, \xi}$ is not an isomorphism on the $\sim P$ part of the $c_{\nu, 0}$ and $c_{\xi, 0}$ equivalence classes. We will do the argument just for the case (a) when $\eta=\xi$; the other cases are similar. It is thus necessary and sufficient to show that for any $\theta(a, x)$ such that $\exists x \theta(a, x) \in \Gamma_{\eta, 1}(a)$,

$$
\Gamma_{\eta, 1}(a) \nvdash \forall x\left[\theta(a, x) \rightarrow \Gamma_{\eta, 0}(x)\right] .
$$


Fix such a $\theta(a, x)$. By elimination of quantifiers, assume without loss that $\theta$ is quantifier-free. If

$$
\Gamma_{\eta, 1}(a) \vdash \exists x[\theta(a, x) \wedge \sim P(x)],
$$

then we are done. Assuming otherwise, it follows by the axioms in 8-10 and 15 that

$$
\Gamma_{\eta, 1}(a) \vdash \forall x[\theta(a, x) \rightarrow \sim(a \leq x)] .
$$

Let $i$ be the largest integer such that some ' $c_{\delta, i}$ ' occurs in $\theta$. If

$$
\Gamma_{\eta, 1}(a) \vdash \exists x\left[\theta(a, x) \wedge \sim\left(c_{\eta, i}<x\right)\right],
$$

then again we are done. Assuming otherwise, it is now routine to prove by induction on the length of $\theta$ that

$$
T^{\prime} \cup\left\{\sim P(a),\left(c_{\eta, j}<a\right), \theta(a, x),\left(g\left(c_{\eta, i+1}, x\right)<c_{\eta, i+1}\right) \mid j<\omega\right\}
$$

is consistent. But then since $T$ is the model completion of $T^{\prime}$ it follows that

$$
\Gamma_{\eta, 1}(a) \vdash \exists x\left[\theta(a, x) \wedge\left(g\left(c_{\eta, i+1}, x\right)<c_{\eta, i+1}\right)\right] .
$$

Therefore

$$
\Gamma_{\eta, 1}(a) \vdash \exists x\left[\theta(a, x) \wedge \sim\left(c_{\eta, i+1}<x\right)\right]
$$

and so we have the desired conclusion. The proof of iii) is now routine.

Note that, by Lemma 5(iii), $T$ has only countably many complete types. Therefore every complete consistent expansion of $T$ in finitely many constant symbols has a prime model. Let $\left\langle\mathfrak{C}_{\xi}, a\right\rangle$ be the prime model of $\Gamma_{\xi, 1}(a)$, where $\mathfrak{C}_{\xi}$ is a model of $T, \xi \in \operatorname{Tr}$. Note that $\mathfrak{C}_{\xi}$ is in fact homogeneous, essentially by the previous lemma. In particular, $\mathfrak{C}_{\xi}$ has only one realization of $\Gamma_{\xi, 1}$. Otherwise, by the axioms for $g$ and axiom $15, \Gamma_{\xi, 0}(x)$ would also be realized in $\mathfrak{C}_{\xi}$, and thus $\Gamma_{\xi, 0}(x)$ would be principal in the theory $\Gamma_{\xi, 1}(a)$, contradicting Lemma 6(ii)(a).

Lemma 7. For every branch $h \in \mathrm{Tr}$, there is a countable homogeneous model $\mathfrak{C}$ of $T$ whose nonprincipal 1-type spectrum is $\left\{\Gamma_{\xi, 1} \mid \xi<h\right\}$. Moreover, $h$ can be computed from the double jump of the satisfaction predicate for $\mathfrak{C}$.

Proof. Let $\mathfrak{C}$ be the union of $\left\{\mathfrak{C}_{\xi} \mid \xi<h\right\}$, where as before $\left\langle\mathfrak{C}_{\xi}, a\right\rangle$ is the prime model of $\Gamma_{\xi, 1}(a)$. Since these are prime models over the appropriate expansion, Lemma 6(i) proves that this set is an elementary chain. Therefore $\mathfrak{C}$ is a model of $T$. Moreover, since each $\mathfrak{C}_{\xi}$ is homogeneous, so is $\mathfrak{C}$. $\mathfrak{C}$ has the desired nonprincipal 1-type spectrum. The last assertion of the corollary follows from the observation that

$$
\begin{aligned}
& h(m)=n \text { iff } \exists d \in|\mathfrak{C}| \forall i<\omega\left[\mathfrak{C} \vDash c_{\eta, i}<d\right], \quad \text { where } \eta=\langle h(0), \cdots, \\
& h(m-1), n\rangle \text {. }
\end{aligned}
$$

Thus $h$ can be defined inductively effectively in an oracle that can compute predicates that are $\Sigma_{2}^{0}$ in the satisfaction predicate of $\mathfrak{C}$. 
Corollary. $T$ has a countable homogeneous model that is not decidable in $A$. Proof. This follows from the previous lemma and the choice of $\operatorname{Tr}$ to have a branch $f$ that was not Turing reducible to $A^{\prime \prime}$.

Lemma 8. (i) If $\mathfrak{B}$ realizes $\Gamma_{\eta, 0}$, then it realizes $\Gamma_{\xi, k}$, for $\xi$ such that $\operatorname{lh}(\xi)<$ $\ln (\eta)$ and $k<2$;

(ii) if $\mathfrak{B}$ realizes $\Gamma_{\xi, 0}$ and has a <-least realization of $\Gamma_{\eta, 0}$, then $\mathfrak{B}$ has a $<$-least realization of $\Gamma_{\xi, 0}$;

(iii) if a model $\mathfrak{B}$ realizes $\Gamma_{\xi, 1}$ and $\Gamma_{\eta, 1}$, where $\xi$ and $\eta$ are incomparable, then $\mathfrak{B}$ realizes $\Gamma_{\delta, k}$ for all $\delta, \ln (\delta) \leq \max \{\operatorname{lh}(\xi), \ln (\eta)\}, k<2$.

Proof. The case for $k=0$ and $\xi<\eta$ in (i) is just Lemma 6(i). The case $k=0, \sim(\xi<\eta)$, and $\operatorname{lh}(\xi)<\operatorname{lh}(\eta)$ then follows by the axioms in 23-25 and the assumption that $\operatorname{Tr}$ has no terminal nodes. For $k=1$, note that it is consistent with the theory $T^{\prime}$ that every realization of $P$ have a $<-$ larger $\sim P$-realization <-above it. Therefore, since $T$ is the model completion of $T^{\prime}$, it follows that

$$
T \vdash \forall x \exists y[P(x) \rightarrow x<y \wedge \neg P(y)] .
$$

The result then follows by the transitivity of $<$.

For (ii), if $\operatorname{lh}(\xi)=\operatorname{lh}(\eta)$ then the result follows by the axioms in 24-26. By the same axioms, it is now enough to consider the case when $\xi$ and $\eta$ are comparable. Assume first that $\operatorname{lh}(\xi)<\operatorname{lh}(\eta)$. Let $c$ be the <-least realization of $\Gamma_{\eta, 0}$ in $\mathfrak{B}$. By the axioms in $19-21, F_{\eta, \xi}(c)$ realizes $\Gamma_{\xi, 0}$ in $\mathfrak{B}$. In order to obtain a contradiction, assume that $F_{\eta, \xi}(c)$ is not the <-least realization of $\Gamma_{\xi, 0}$. Let $b \in|\mathfrak{B}|$ be a realization of $\Gamma_{\xi, 0}$ satisfying $\mathfrak{B} \vDash b<F_{\eta, \xi}(c)$. The following is consistent:

$$
T^{\prime} \cup \Delta_{\mathfrak{B}} \cup\left\{F_{\eta, \xi}(x)=b, x<c\right\},
$$

where $\Delta_{\mathfrak{B}}$ is the atomic diagram of $\mathfrak{B}$. Therefore, since $T$ is the model completion of $T^{\prime}, \exists d \in|\mathfrak{B}|$ satisfying

$$
\mathfrak{B} \vDash\left[F_{\eta, \xi}(d)=b \wedge d<c\right] \text {. }
$$

Since $d$ is <-less than $c$, it must be <-comparable with all of the $c_{\xi, i}$ 's. Since $c$ was chosen to be the <-least realization of $\Gamma_{\xi, 0}$, there exists an $i<\omega$ such that $\mathfrak{B} \vDash d<c_{\xi, i}$. Therefore by the axioms in 19 and 20 ,

$$
\mathfrak{B} \vDash F_{\eta, \xi}(d)<c_{\xi, i} .
$$

But this contradicts the choice of $b$.

Next assume that $\ln (\eta)<\ln (\xi)$. Let $c$ be the <-least realization of $\Gamma_{\eta, 0}$ in $\mathfrak{B}$. By the same argument involving $T^{\prime}$ as above, $\exists b \in|\mathfrak{B}|$ satisfying $\mathfrak{B} \vDash F_{\xi, \eta}(b)=c$. Assume in order to obtain a contradiction that $b$ is not the <-least realization of $\Gamma_{\xi, 0}$ in $\mathfrak{B}$. Let $d$ be a realization of $\Gamma_{\xi, 0}$ that is <-less than $b$. Then by the axioms in $19-20, F_{\xi, \eta}(d)$ realizes $\Gamma_{\xi, 0}$ in $\mathfrak{B}$ and is $<-$ less than $c$, a contradiction. 
For (iii) assume first that $\operatorname{lh}(\xi)=\operatorname{lh}(\eta)$. Let $b$ and $c$ realize $\Gamma_{\eta, 1}$ and $\Gamma_{\xi, 1}$ in $\mathfrak{B}$ respectively. Then by the axioms in $30-33, G_{\eta, \xi}(b, c)$ realizes $\Gamma_{\xi, 0}$ in $\mathfrak{B}$. Thus $\Gamma_{\delta, k}$ is realized for all $\delta, \operatorname{lh}(\delta)<\operatorname{lh}(\xi)$, by (i).

Assume next that say $\operatorname{lh}(\xi)<\operatorname{lh}(\eta)$. Lemma 6(i) and the same argument as above applied to $\delta=\left.\eta\right|_{\ln (\xi)}$ show that $\Gamma_{\delta, 0}$ is realized in $\mathfrak{B}$. Let $b$ and $c$ realize $\Gamma_{\eta, 1}$ and $\Gamma_{\delta, 0}$ in $\mathfrak{B}$ respectively. It is straightforward to check that $g\left(F_{\eta, \delta}(b), c\right)$ realizes $\Gamma_{\delta, 0}$ in $\mathfrak{B}$. The usual argument then shows that there must be $d \in|\mathfrak{B}|$ satisfying

$$
\mathfrak{B} \vDash\left[d<b \wedge F_{\eta, \delta}(d)=g\left(F_{\eta, \delta}(b), c\right)\right] .
$$

Since $d$ is <-less than $b$, it must be <-comparable with the $c_{\eta, i}$ 's. But if it were <-less than $c_{\eta, i}$ for some $i$, then $g\left(F_{\eta, \delta}(b), c\right)$ would have to be <-less than $c_{\delta, i}$, contradicting its realizing $\Gamma_{\delta, 0}$. Therefore $d$ must be <-larger than all the $c_{\delta, i}$ 's, and so must realize $\Gamma_{\eta, 0}$.

By the previous three lemmas, any model $\mathfrak{C}$ of $T$ must satisfy exactly one condition from:

A. $\mathfrak{C}$ omits $\Gamma_{\langle\rangle, 1}$;

B. there is some $f \in 2^{<\omega} \cup 2^{\omega}$ such that $\mathfrak{C}$ omits $\Gamma_{\delta, 0}$, for all $\delta$, and realizes $\Gamma_{\xi, 1}$ iff $\xi \leq f$;

C. there is some $n \leq \omega$ such that $\mathfrak{C}$ has a <-least realization of $\Gamma_{\xi, 0}$ iff $\operatorname{lh}(\xi)<n$, and $\mathfrak{C}$ omits $\Gamma_{\eta, k}, \operatorname{lh}(\eta) \geq n, k<2$;

D. there is some $n \leq \omega$ such that $\mathfrak{C}$ realizes $\Gamma_{\xi, 0}$ iff $\operatorname{lh}(\xi)<n$, but there are no such realizations that are <-least, and $\mathfrak{C}$ omits $\Gamma_{\eta, k}, \operatorname{lh}(\eta) \geq n$, $k<2$.

Lemma 9. If two countable models of $T$ realize the same option from A-D above, and have the same number of equivalence classes in their respective realizations of $\Gamma_{\omega, 0}$, then they are isomorphic.

Proof. The axioms of $T^{\prime}$ make it easy to see that if $C, D$ are equivalence classes of countable models of $\mathfrak{U}, \mathfrak{B}$ respectively such that elements of $C, D$ realize $\Gamma_{\omega, k}$, then $\left.\left.\mathfrak{A}\right|_{C} \cong \mathfrak{B}\right|_{D}$. Because the function symbols all have range $\left\{c_{\langle\rangle, 0}\right\}$ on $C, D$, it is also easy to see that if

$$
\left.\left.\mathfrak{A}\right|_{|\mathfrak{A}|-C} \cong \mathfrak{B}\right|_{|\mathfrak{B}|-D},
$$

then any isomorphism of $\left.\mathfrak{A}\right|_{C},\left.\mathfrak{B}\right|_{D}$ can be extended to an isomorphism of $\mathfrak{A}$ and $\mathfrak{B}$. So it is enough to consider the restriction of countable models $\mathfrak{C}$ of $T$ to those elements not realizing $\Gamma_{\omega, k}$ for either $k=0$ or $k=1$. Let us refer to that restriction of such a $\mathfrak{C}$ as the $\Gamma_{<\omega^{-}}$restriction of $\mathfrak{C}$. If two models omit $\Gamma_{\langle\rangle, 0}$, then by the previous lemmas their $\Gamma_{<\omega^{-}}$restrictions are prime and therefore isomorphic. If two countable models satisfy $D$ for the same $n$, then it easy to check that the $\Gamma_{<\omega^{-}}$restrictions are homogeneous and have the same type spectrum. Therefore they are isomorphic. Any two countable $\Gamma_{<\omega^{-}}$ 
restrictions satisfying $B$ for the same $f$ can be expressed as the union of a chain of reducts of prime models of $\Gamma_{\xi, 1}, \xi<f$, and are therefore isomorphic. Similarly any two countable $\Gamma_{<\omega^{-}}$restrictions satisfying $C$ for the same $n$ can be expressed as the union of the reducts of the prime models of the same $\Gamma_{\xi, 0}$ 's, and are therefore isomorphic.

Corollary. $T$ has only countably many countable models up to isomorphism.

Proof. This follows by the previous lemma and the choice of $\operatorname{Tr}$ to have only countably many branches.

It remains to prove

Lemma 10. $T^{\prime}$ has a complete, decidable, model completion $T$.

Proof. Let $L_{1}$ be the language $L$ with only the nonlogical symbols

$$
\left\{c_{\xi, i}, E,<, F_{\xi, \eta}, g \mid \xi<\eta \in \operatorname{Tr}, i<\omega\right\} .
$$

In [4] a universal theory is constructed that has a complete, decidable, model completion, and the universal theory contains the axioms of $T^{\prime}$ that relate to the symbols in $L_{1}$. An examination of the proof of the existence of such a model completion shows that for any recursive $\operatorname{Tr} \subseteq 2^{<\omega}$ and theory $T_{1}^{\prime}$ whose axioms are 1-14, 17-20, and 29 from above with respect to Tr, the theory $T_{1}^{\prime}$ has a complete, decidable, model completion. We will now define a model $\mathfrak{D}$ of the desired complete, decidable, model completion $T$ of our $T^{\prime}$. Let $T_{1}$ be the complete, decidable, model completion of the axioms in 1-14, 17-20, and 29 , with respect to

$$
\operatorname{Tr}^{\prime}:=\left\{\alpha \in 2^{<\omega} \mid \alpha<F\right\},
$$

where $F(n)=0$ for all $n$. By the results in [4], the types $\Gamma(x)$ and $\Sigma_{\eta}(y)$ containing the formulas

$$
\left\{\sim E\left(x, c_{\xi, 0}\right) \mid \xi<F\right\}, \quad\left\{c_{\eta, i}<y \mid i<\omega\right\}, \quad \text { respectively }
$$

are nonprincipal, $\eta<F$. Fix a countable model $\mathfrak{A} \vDash T_{1}$ omitting these types. Define

$$
\begin{gathered}
H:=\left\{\langle a, \xi\rangle|a \in| \mathfrak{A} \mid, \exists i \exists \eta\left[\langle U, a\rangle \vDash \exists\left(C_{\eta}, i, a\right), \operatorname{lh}(\eta)=\operatorname{lh}(\xi)\right], \xi \in \operatorname{Tr}\right\} . \\
|\mathfrak{B}|:=\left\{b_{a, \eta} \mid\langle a, \eta\rangle \in H\right\} .
\end{gathered}
$$


Next define

$$
\begin{aligned}
& E_{\mathfrak{B}}:=\left\{\left\langle b_{a, \eta}, b_{d, \eta}\right\rangle \mid\langle a, \eta\rangle,\langle d, \eta\rangle \in H\right\} ; \\
& \left(c_{\eta, i}\right)_{\mathfrak{B}}:=b_{a, \eta}, \text { where } \exists\langle a, \xi\rangle \in H\left[\ln (\xi)=\operatorname{lh}(\eta) \text { and } a=\left(c_{\xi, i}\right)_{\mathfrak{A}}\right] \\
& <_{\mathfrak{B}}:=\left\{\left\langle b_{a, \eta} b_{d, \eta}\right\rangle \mid\langle a, d\rangle \in\left(<_{\mathfrak{A}}\right),\langle a, \eta\rangle,\langle d, \eta\rangle \in H\right\} ; \\
& g_{\mathfrak{B}}\left(b_{a, \eta}, b_{d, \eta}\right):=b_{e, \eta}, \text { where } g_{\mathfrak{A}}(a, d)=e \neq\left(c_{\langle\rangle, 0}\right)_{\mathfrak{A}} ; \\
& g_{\mathfrak{B}}(x, y):=c_{\langle\rangle, 0} \text { for all } x, y \in|\mathfrak{B}| \text { not covered by the last case; } \\
& \left(I_{\eta, \delta}\right)_{\mathfrak{B}}\left(b_{a, \eta}\right):=b_{a, \delta} ; \\
& \left(I_{\eta, \delta}\right)_{\mathfrak{B}}(a):=c_{\langle\rangle, 0} \text { for all } a, \eta, \delta \text { not covered in the previous case; } \\
& \left(F_{\eta, \delta}\right)_{\mathfrak{B}}\left(b_{a, \eta}\right):=b_{d, \delta} \text { where }\left(F_{\eta, \delta}\right)_{\mathfrak{A}}(a)=d ; \\
& \left(F_{\eta, \delta}\right)_{\mathfrak{B}}\left(b_{a, \eta}\right):=c_{\langle\rangle, 0} \text { for all } b, \eta, \delta \text { not covered in the previous case; } \\
& G_{\mathfrak{B}}(x, y):=c_{\langle\rangle, 0} \text { for all } x, y \in|\mathfrak{B}| ; \\
& P_{\mathfrak{B}}:=|\mathfrak{B}| .
\end{aligned}
$$

It is routine to check that $\mathfrak{B}$ satisfies all the axioms of $T^{\prime}$. Let the set $V$ contain exactly one element of each $E$-equivalence class of $\mathfrak{B}$. For each $v \in V$, let $\operatorname{Br}_{v}$ be a countable set of functions $f: \omega \rightarrow\left\{b \mid\langle b, v\rangle \in E_{\mathfrak{B}}\right\}$ such that:

(i) $\forall v \in V \forall f \in \mathrm{Br}_{v} \forall i<j\left[\langle f(i), f(j)\rangle \in\left(<_{\mathfrak{B}}\right)\right]$;

(ii) $\forall v \in V \forall f \in \mathrm{Br}_{v} \forall b \in|\mathfrak{B}| \exists i\left[\langle f(i), b\rangle \notin\left(<_{\mathfrak{B}}\right)\right]$;

(iii) $\forall v \in V \forall b \in|\mathfrak{B}| \exists f \in \mathrm{Br}_{v} \exists i$ [if $\langle b, v\rangle \in E_{\mathfrak{B}}$ then $\left.\langle b, f(i)\rangle \in\left(<_{\mathfrak{B}}\right)\right]$;

(iv) $\forall v \in V \forall f, h \in \mathrm{Br}_{v} \exists i\left[\langle f(i), h(i)\rangle,\langle h(i), f(i)\rangle \notin\left(<_{\mathfrak{B}}\right)\right]$;

(v) $\forall \xi<\eta \forall u, v \in V \forall f \in \mathrm{Br}_{u}$ [if $\left\langle u, c_{\eta, 0}\right\rangle,\left\langle v, c_{\xi, 0}\right\rangle \in E_{\mathfrak{B}}$ then $F_{\eta, \xi} \circ f \in$ $\left.\mathrm{Br}_{v}\right]$;

(vi) $\forall \xi, \eta \forall u, v \in V \forall f \in \mathrm{Br}_{u}$ [if $\operatorname{lh}(\eta)=\operatorname{lh}(\xi),\left\langle u, c_{\eta, 0}\right\rangle,\left\langle v, c_{\xi, 0}\right\rangle \in E_{\mathfrak{B}}$ then $I_{\eta, \xi} \circ f \notin \mathrm{Br}_{v}$;

(vii) $\forall \xi<\eta \forall u \in V \forall f \in \mathrm{Br}_{u} \forall i \forall b \in|\mathfrak{B}|$ \{[if there is a $j<\omega$ such that $\left.\left.\langle\mathfrak{B}, f(j), b\rangle \vDash\left(c_{\xi, i}<f(j)\right) \wedge\right\rceil\left(c_{\eta_{i+1}}<b\right)\left(c_{\eta, i}<b\right)\right]$ then $\exists v \in V \exists h \in$ $\mathrm{Br}_{v} \exists j\left[\langle\mathcal{B}, h(j)\rangle \vDash(b<h(j))\right.$ and $\left.f=F_{\eta, \xi} \circ h\right]$.

We will now define the model $\mathfrak{D}$ in terms of $\mathfrak{B}$ and $\mathrm{Br}_{v}$. Each set $\mathrm{Br}_{v}$ is, loosely speaking, a dense set of branches in the $E$-equivalence class of $v$. The reason that $\operatorname{Th}(\mathfrak{B})$ is not the desired theory is that it has no realizations of $\sim P$, and so therefore is not model consistent with $T^{\prime}$. To remedy this we will now add realizations of $\sim P$. In order to be model consistent with $T^{\prime}$, the realizations of $\sim P$ must be "upwardly dense", in other words <-above every element there must be a realization of $\sim P$. Thus we will append a realization of $\sim P$ at the "end" of each branch $f \in \mathrm{Br}_{v}, v \in V$. And of course we must attend to the behavior and density of the $\sim P$-realizations with respect to the 
various functions. Therefore define

$$
\begin{aligned}
& |\mathfrak{D}|:=|\mathfrak{B}| \cup\left\{d_{v, f} \mid f \in \mathrm{Br}_{v}, v \in V\right\} ; \\
& E_{\mathfrak{D}}:=E_{\mathfrak{B}} \cup\left\{\left\langle d_{v, f}, b\right\rangle,\left\langle b, d_{v, f}\right\rangle \mid\langle v, b\rangle \in E_{\mathfrak{B}}, f \in \mathrm{Br}_{v}, v \in V\right\} \\
& \quad \cup\left\{\left\langle d_{v, f}, d_{v, h}\right\rangle \mid f, h \in \mathrm{Br}_{v}, v \in V\right\} ; \\
& \left(c_{\xi, i}\right)_{\mathfrak{D}}:=\left(c_{\xi, i}\right)_{\mathfrak{B}} ; \\
& <_{\mathfrak{D}}:=<_{\mathfrak{B}} \cup\left\{\left\langle b, d_{v, f}\right\rangle\left|\exists i\left[\langle b, f(i)\rangle \in<_{\mathfrak{B}}\right], f \in \mathrm{Br}_{v}, v \in V, b \in\right| \mathfrak{B} \mid\right\} ; \\
& g_{\mathfrak{D}}\left(b, b^{\prime}\right):=g_{\mathfrak{B}}\left(b, b^{\prime}\right) \quad \text { for } \quad b, b^{\prime} \in|\mathfrak{B}| ; \\
& g_{\mathfrak{D}}\left(d_{v, f}, b\right):=g_{\mathfrak{D}}\left(b, d_{v, f}\right):=\operatorname{Lim}_{s \rightarrow \infty} g_{\mathfrak{B}}(f(s), b) \\
& \quad \text { for } \quad f \in \mathrm{Br}_{v}, v \in V, b \in|\mathfrak{B}| ; \\
& g_{\mathfrak{D}}\left(d_{v, f}, d_{w, h}\right):=\operatorname{Lim}_{s \rightarrow \infty} g_{\mathfrak{B}}(f(s), h(s)) \quad \text { for } \quad f \in \mathrm{Br}_{v}, h \in \mathrm{Br}_{w}, v, w \in V ; \\
& \left(I_{\xi, \eta}\right)_{\mathfrak{D}}(b):=\left(I_{\xi, \eta}\right)_{\mathfrak{B}}(b) \quad \text { for } \quad b \in|\mathfrak{B}| ; \\
& \left(I_{\xi, \eta}\right)_{\mathfrak{D}}\left(d_{v, f}\right):=c_{\langle\rangle, 0} \text { for } \quad f \in \mathrm{Br}_{v}, v \in V ; \\
& \left(F_{\xi, \eta}\right)_{\mathfrak{D}}(b):=\left(F_{\xi, \eta}\right)_{\mathfrak{B}}(b) \quad \text { for } \quad b \in|\mathfrak{B}| ; \\
& \left(F_{\xi, \eta}\right)_{\mathfrak{D}}\left(d_{v, f}\right):=d_{w, h} \quad \text { where } \quad\left\langle v, c_{\xi, 0}\right\rangle,\left\langle w, c_{\eta, 0}\right\rangle \in E_{\mathfrak{B}} \quad \text { and } \quad h=F_{\xi, \eta} \circ f ; \\
& \left(G_{\xi, \eta}\right)_{\mathfrak{D}}(b, d):=\left(G_{\xi, \eta}\right)_{\mathfrak{D}}(d, b):=c_{\langle\rangle, 0} \quad \text { for } \quad b \in|\mathfrak{B}|, d \in|\mathfrak{D}| ; \\
& \left(G_{\xi, \eta}\right)_{\mathfrak{D}}\left(d_{v, f}, d_{w, h}\right):=\operatorname{Lim}_{s \rightarrow \infty} g_{\mathfrak{B}}\left(I_{\xi, \eta}(f(s)), h(s)\right) \\
& P_{\mathfrak{D}}:=\left\{d_{v, f} \mid f \in \mathrm{Br}_{v}, v \in V\right\} . \quad \text { for } \quad f \in \mathrm{Br}_{v}, h \in \mathrm{Br}_{w}, v, w \in V ;
\end{aligned}
$$

Let $T:=\operatorname{Th}(\mathfrak{D})$. It is straightforward to check that $T^{\prime}$ and $T$ are mutually model consistent. We will sketch the main details necessary to show that $T$ allows elimination of quantifiers. Let $\theta(\bar{x}, \bar{y})$ be a quantifier-free formula in the displayed free variables such that $T \cup\{\theta(\bar{x}, \bar{y})\}$ is consistent. We must show that there exists a quantifier-free $\psi(\bar{y})$ such that $T \vDash \forall \bar{y}[\exists \bar{x} \theta(\bar{x}, \bar{y}) \leftrightarrow \psi(\bar{y})]$. For any $N<\omega$ let $L_{N}$ be the language of $T$ restricted to the nonlogical symbols

$$
P,<, g, E, c_{\eta, i}, F_{\xi, \eta}, G_{\xi, \eta} \text {, or } I_{\xi, \eta}, \quad \text { where } i, \operatorname{lh}(\xi), \operatorname{lh}(\eta) \leq N \text {. }
$$

Fix an $N$ such that $\theta$ is a formula in ${ }^{\prime} L_{N}$. Let $\mathfrak{H}$ be the set of all maximal subsets $M$ of atomic and negated atomic formulas from $L_{N}$ in the variables from $\operatorname{rg}(\bar{x}) \cup \operatorname{rg}(\bar{y})$, such that

$$
T \cup M \cup\{\theta(\bar{x}, \bar{y})\} \text { is consistent. }
$$

Since $L_{N}$ is finite, there are at most finitely many such $M$. By Lemma 1 , for each such $M$ there is a finite $M^{\prime} \subseteq M$ such that $T \cup M^{\prime} \vdash \phi(\bar{x}, \bar{y})$ for all $\phi(x, \bar{y}) \in(M \cup\{\theta(x, \bar{y})\})$. And thus we have that

$$
T \vdash \forall \bar{x} \forall \bar{y}\left[\theta(\bar{x}, \bar{y}) \leftrightarrow \bigvee_{M \in \mathfrak{J}}\left[\bigwedge_{\phi \in M^{\prime}} \phi(\bar{x}, \bar{y})\right]\right.
$$


It is thus enough to show that for some such $M^{\prime}$, the result holds if $\theta$ is actually of the form $\bigwedge_{\phi \in M^{\prime}} \phi(\bar{x}, \bar{y})$. So fix such an $M$ and $M^{\prime}$. We will assume that for every term $\tau$ such that ' $\tau$ ' occurs in our new $\theta$, there is a $z \in \operatorname{rg}(\bar{y}) \cup \operatorname{rg}(\bar{x})$ such that

$$
T \cup M^{\prime} \vdash z=\tau .
$$

Of course this is in general not the case, but by adding extra variables to $\bar{x}$ if necessary, we can make this assumption without loss of generality. Also without loss we will make two other assumptions about the form of the formulas in $M^{\prime}$ :

(i) if $\tau$ is a term that is not a variable or constant symbol, and ' $\tau$ ' occurs in some $\phi \in M^{\prime}$, then $\phi$ is of the form ' $\tau=z$ ' for some $z \in \operatorname{rg}(\bar{x}) \cup \operatorname{rg}(\bar{y})$, and no other formula in $M^{\prime}$ contains an occurrence of ' $\tau$ ';

(ii) if $T \cup M \vdash(x=y)$ for some $x \in \operatorname{rg}(\bar{x})$ and $y \in \operatorname{rg}(\bar{y})$, then $(x=y) \in$ $M^{\prime}$, and no other formula in $M^{\prime}$ contains an occurrence of ' $y$ '.

Now let $M^{\prime \prime} \subseteq M^{\prime}$ be those formulas whose nonlogical symbols are in $L\left(T_{1}\right)$. Since $T_{1}$ allows elimination of quantifiers, there is a quantifier-free formula $\psi_{1}(\bar{y})$ of $L\left(T_{1}\right)$ such that

$$
T_{1} \vdash \forall \bar{y}\left[\exists \bar{x}\left(\bigwedge_{\phi \in M^{\prime \prime}} \phi(\bar{x}, \bar{y})\right) \leftrightarrow \psi_{1}(\bar{y})\right] .
$$

Again by Lemma 1 , there is a finite $M^{\prime \prime} \subseteq M$ such that no formula in $M^{\prime \prime}$ has an occurrence of any ' $x$ ' for $x \in \operatorname{rg}(\bar{x})$, and $T \cup M^{\prime}$ ' $\phi(\bar{y})$ for each $\phi(\bar{y}) \in M$ that has no occurrence of any ' $x$ ' for $x \in \operatorname{rg}(\bar{x})$. Define

$$
\psi(\bar{y}):=\psi_{1}(\bar{y}) \wedge\left[\bigwedge_{\phi \in M^{\prime \prime}} \phi(\bar{y})\right] .
$$

The claim is that this is the desired $\psi$. By all of the choices above it is clear that

$$
T \vdash \forall \bar{y}[\exists \bar{x} \theta(\bar{x}, \bar{y}) \rightarrow \psi(\bar{y})] .
$$

Next fix a $\mathfrak{U} \vDash T$ and $\bar{a} \in|\mathfrak{U}|^{\langle\omega}$ such that $\langle\mathfrak{U}, \bar{a}\rangle \vDash \psi(\bar{a})$. Also fix the largest $N$, then $\xi \in 2^{N}$ and an $a \in \operatorname{rg}(\bar{a})$ such that $\langle\mathfrak{U}, a\rangle \vDash E\left(c_{\xi,\langle\rangle}, a\right)$. This is possible by our choice of $\mathfrak{D}$. Define

$$
V:=\left\{a \in \operatorname{rg}(\bar{a}) \mid\langle\mathfrak{U}, a\rangle \vDash E\left(c_{\xi,\langle\rangle}, a\right)\right\} .
$$

Since $\mathfrak{U} \vDash T_{1}$, and $\langle\mathfrak{U}, \bar{a}\rangle \vDash \psi_{1}(\bar{a})$, it follows that there is a $\bar{b} \in|\mathfrak{U}|^{<\omega}$ such that

$$
\langle\mathfrak{U}, \bar{a}, \bar{b}\rangle \vDash\left(\bigwedge_{\phi \in M^{\prime \prime}} \phi(\bar{b}, \bar{a})\right) .
$$

Fix the largest $N^{\prime}$, then a $\gamma \in 2^{N^{\prime}}$, where $\gamma$ and $\xi$ are comparable, and a $b \in \operatorname{rg}(\bar{b})$ such that $\langle\mathfrak{U}, b\rangle \vDash E\left(c_{\gamma,\langle\rangle}, b\right)$. If there is no such $N^{\prime}$, then by choice of $\mathfrak{D}$ the result is immediate. Now define

$$
V^{\prime}:=\left\{b \in \operatorname{rg}(\bar{b}) \mid\langle u, b\rangle \vDash E\left(c_{\gamma,\langle\rangle}, b\right)\right\} .
$$

Next let $V=\left\{a_{i_{j}} \mid 1 \leq j \leq m\right\}$, where $\bar{a}=\left\langle a_{1}, a_{2}, \ldots\right\rangle$, and let $V^{\prime}=$ $\left\{b_{i_{j}} \mid 1 \leq j \leq m^{\prime}\right\}$, where $\bar{b}=\left\langle b_{1}, b_{2}, \ldots\right\rangle$. Then by the assumptions on $\theta$ it 
follows that for every $x \in \operatorname{rg}(\bar{x})$ there is some term $\tau$ whose only variables are from $\left\{y_{i_{j}} \mid 1 \leq j \leq m\right\} \cup\left\{x_{i_{j}} \mid 1 \leq j \leq m^{\prime}\right\}$, and such that $T \cup M$ F $(\tau=x)$.

Let $\bar{x}=\left\langle x_{1}, x_{2}, \ldots, x_{n}\right\rangle$ and for each $k, 1 \leq k \leq n$, fix $\tau_{k}$ whose only variables are from $\left\{y_{i_{j}} \mid 1 \leq j \leq m\right\} \cup\left\{x_{i_{j}} \mid 1 \leq j \leq m^{\prime}\right\}$ and such that $T \cup M$ F $\left(\tau=x_{k}\right)$. Define $\bar{c}:=\left\langle c_{1}, c_{2}, \ldots, c_{n}\right\rangle$ where

$$
c_{k}=\tau_{k}\left(\frac{x_{i_{1}}}{b_{i_{1}}}, \frac{x_{i_{1}}}{b_{i_{2}}}, \ldots, \frac{x_{i_{m^{\prime}}}}{b_{i_{m^{\prime}}}}, \frac{y_{i_{1}}}{a_{i_{1}}}, \frac{y_{i_{2}}}{a_{i_{2}}}, \ldots, \frac{y_{i_{m}}}{a_{i_{m}}}\right) .
$$

It is then routine to check that $\langle\mathfrak{U}, \bar{a}, \bar{c}\rangle \vDash \theta(\bar{c}, \bar{a})$, and so $\langle\mathfrak{U}, \bar{a}\rangle \vDash \exists \bar{x} \theta(\bar{x}, \bar{a})$. Therefore

$$
T \vdash \forall \bar{y}[\exists \bar{x} \theta(\bar{x}, \bar{y}) \leftrightarrow \psi(\bar{y})] .
$$

Since $T_{1}$ is decidable, it is easy to see that the above procedure for eliminating quantifiers is effective and so $T$ is decidable.

\section{REFERENCES}

1. T. Millar, Foundations of recursive model theory, Ann. Math. Logic 13 (1978), 45-72.

2. __ Decidability and the number of countable models, Ann. Pure Appl. Logic 27 (1984), 137-153.

3. _ Type structure complexity and decidability, Trans. Amer. Math. Soc. 272 (1981), 73-81.

4. __ Bad models in nice neighborhoods, J. Symbolic Logic 51 (1986), 1043-1056.

5. H. Rogers, Jr., Theory of recursive functions and effective computability, McGraw-Hill, 1967.

Department of Mathematics, University of Wisconsin, Madison, Wisconsin 53706 